\title{
Evaluation of the accuracy of working length determination and automatic apical reverse function accuracy of endodontic rotary motor integrated apex locator: an in-vitro study
}

\author{
Nanda Kishore K1,*, Madhu Varma K², Girija S Sajjan², Kalyan Satish R², Raheem Md1, \\ Anitha Viswanadhan ${ }^{3}$ \\ ${ }^{1}$ Postgraduate Student,, ${ }^{2}$ Professor, ${ }^{3}$ Senior Lecturer, Department of Conservative Dentistry and \\ Endodontics, Vishnu Dental College, Bhimavaram-534202, West Godavari, Andhra Pradesh, India.
}

\section{N F O R M A T I O N}

\section{Article History}

Received $2^{\text {nd }}$ January 2020

Accepted 31 st January 2020

Available online

$9^{\text {th }}$ March 2020

\section{K E Y W O R D S}

Automatic apical reverse

Electronic apex locator

Endodontic motor

Working Length
A B S T R A C T

Background: The outcome of a Root canal therapy depends upon complete cleaning of the root canal system without damaging periapical integrity. Accurate determination of working length determination is essential. The development of Endodontic motor integrated apex locators (EALs) for locating canal terminus has been significant innovation in the field of Endodontics.

Aim: To evaluate the accuracy of Endomotor integrated electronic apex locator in determining the working length before and after cleaning and shaping with Automatic Apical Reverse action set at the "0.5mm" mark.

Materials and methods: Forty extracted premolars were decoronated, patency was verified. The actual length of tooth measured and teeth were embedded in alginate and file was advanced. Readings at the apex and $0.5 \mathrm{~mm}$ short of the apex was recorded using Dentaport ZX (DZ group) and EConnect S (ES group) apex locator. Cleaning and shaping were done with rotary files with Automatic Apical Reverse action set at $0.5 \mathrm{~mm}$ short of apex and reading recorded as $\mathrm{Au}$ tomatic Apical Reverse Length (AARL), and actual tooth length is measured. The data obtained were statistically analyzed.

Results: Within the limitations of the study, the EALs readings of the DZ group \& ES group provided an acceptable determination of working length within range $\pm 0.5 \mathrm{~mm}$, and AAR function set at $0.5 \mathrm{~mm}$ mark of Endomotor integrated apex locator (E Connect $S$ ) provided an adequate apical limit.

Conclusion: Under the in vitro conditions of this study, both Electronic Apex Locators showed an acceptable determination of working length within range $\pm 0.5 \mathrm{~mm}$ from the actual length. The AAR function set at the $0.5 \mathrm{~mm}$ mark of Econnect $S$ provided an adequate apical limit.

\section{Introduction}

Root canal therapy (RCT) is the most common treatment for the pulpally diseased teeth; determination of appropriate working length is one of the vital factors for the success of root canal therapy [1]. According to the Glossary of endodontic terms, "the working length (WL) is defined as the distance from a coronal reference point to the point at which canal preparation and obturation 
should terminate". The traditional method of determining the WL was by taking the intraoral periapical radiographs. With the advent of Electronic apex locators (EALs) in the endodontics has helped to make determining the WL more precise, accurate, and predictable [2,3]. Different generations of EALs have been introduced in the market to locate the root apex for measuring the WL. Endodontic motor integrated EAls have been developed, making the root canal therapy faster and simpler $[4,5]$. These hybrid devices not only have the torque and speed control but also aim to monitor and maintain the apical limit during mechanical shaping of root canals [5]. The Automatic Apical Reverse (AAR) function stops and reverses the rotation when the specified point is reached by the file tip. This function allows controlling the apical extent of canal instrumentation. The AAR function can be set at different levels indicating file closer or away from the apical constriction. The AAR function has been set at the "0.5 mm" mark as it corresponds to the location of apical constriction (AC), and the APEX mark corresponds to the tip of the file at apical foramen (AF). The DentaPort ZX (Morita, Tokyo, Japan), is a frequency-dependent device, determines the position of the WL by simultaneous measurement of impedance values in the same canal using two different frequencies (8 and $0.4 \mathrm{kHz}$ ) [6]. The EConnect S (Eighteeth, China) is a multi-frequency based EAL with high precision and accuracy, which can function as low-speed handpiece and apex locator or combination of both. In the present study, Dentaport ZX (DZ group) was used to compare the accuracy of the apex locator alone with $\mathrm{E}$ Connect S (ES group). This in-vitro study aimed to evaluate the accuracy of Endomotor integrated electronic apex locator in determining the working length before and after cleaning and shaping with Automatic Apical Reverse action set at " $0.5 \mathrm{~mm}$ " mark.

\section{Materials and methods}

Forty extracted human premolars were taken, which are free of caries, single-rooted, and with a single canal were selected. Teeth with developmental disturbances, with caries, teeth which are multirooted, calcified, with open apices and presence of extra canal, were excluded from this study. The teeth were decoronated with a diamond disc to obtain a standard length of $15 \mathrm{~mm}$ and to obtain a plain surface. A size 15K file (Dentsply Maillefer, Ballaigues, Switzerland) was used to confirm the patency and presence of a single canal. The root canals were pre flared using SX rotary instruments (Dentsply Sirona) with $2.5 \%$ sodium hypochlorite $(\mathrm{NaOCl})$ as an irrigating solution. The AF standardization and visual measurement were performed under the Dental Operating Microscope until the tip of the $15 \mathrm{k}$ file visible at the most coronal border of AF opening. Then the rubber stop was adjusted to the reference point. The distance between the rubber stop and file tip was measured with a digital caliper (DC) to $0.02 \mathrm{~mm}$ precision and recorded as actual length (AL). The teeth were embedded in a freshly made alginate.

\subsection{Apex locator function}

To evaluate the apex locator function of the devices, the $15 \mathrm{~K}$ file was inserted into the root canal, and the device was connected to the lip clip placed in alginate and the file holder to the $15 \mathrm{~K}$ file. The file was advanced into the root canal until the "APEX" mark and distance between the reference point and file tip was recorded as (EL APEX). Then file withdrawn until the" 0.5 " mm mark of the device to obtain the electronic working length (EL 0.5). The measurements were recorded if the mark displayed remained stable for at least 5 seconds. The electronic measurements of all 40 teeth were obtained with both the apex locators (i.e., Dentaport ZX and E Connect S).

\subsection{AAR function}

The AAR function of the Econnect $\mathrm{S}$ was adjusted to the "0.5" mm mark. The chemomechanical preparation was done with Protaper Gold (Dentsply Sirona) rotary instruments (up to F2) were used until the apical limit was reached, and the AAR was activated. Irrigation was done with $2.5 \% \mathrm{NaOCl}$ following each instrument. The last file F2 was manually inserted into the extent of root canal preparation, and the rubber stop was adjusted to the reference point. The distance between the rubber stop and file tip was measured using a digital calliper and recorded as AAR length, which is also done with the Dentaport ZX apex locator. The teeth were removed from the alginate and washed, and a 25.02 stainless steel file was inserted into the canal up to $\mathrm{AF}$ to measure the actual length after preparation (AL2). All the electronic measurements were performed by a single operator who is unaware of visual measurements.

\subsection{Statistical analysis}

The data obtained were tabulated and statistically analyzed, from the measurements obtained by the EAL and AAR function, the AL and AL2 were subtracted. 
The differences in the electronic readings were assigned as negative and positive, respectively. The Kolmogorov Smirnov test confirmed the normal distribution. The Analysis of variance test used to compare mean root length among measuring methods. The post hoc Tukey test was used to compare the percentage of distribution of the electronic measurements between devices (significance set at 0.05 level).

\section{Results}

The mean AL was $14.54 \mathrm{~mm}$, and the mean length after preparation (AL2) was $14.43 \mathrm{~mm}$; these results were not statistically different $(p=0.12)$. Table 1 presents the descriptive for the electronic measurements according to different functions. There was no significant difference among the two tested devices $(\mathrm{p}=$ 0.926). Tables 2 and 3 presents the EAL and AAR functions, respectively, in terms of the distributions and percentages of the difference between the electronic readings and the visual measurements.

\section{Discussion}

In the present study, the alginate medium was used to simulate the periodontium as it remains around the root, and its colloidal consistency presents a suitable electroconductive medium [7]. The teeth were Decoronated to create a flat, stable reference point to provide consistent measurements. The coronal pre flaring was done as it increases the accuracy of the EALs [8]. The tolerance range of $\pm 0.5 \mathrm{~mm}$ for the EAL is considered acceptable [9]. Even the range of \pm 0.5 $\mathrm{mm}$ might overestimate the precision of EAL, most of the endodontic rulers can measure to an accuracy of $0.5 \mathrm{~mm}$. The EAL measurements for APEX and $0.5 \mathrm{~mm}$ mark showed $100 \%$ within limits for both the Electronic Apex locators. The EALs were compared with Actual length as it is the constant landmark for evaluation, as it is expected that the morphology of AC might be altered during the shaping procedures [10]. The EAL readings beyond $\pm 1 \mathrm{~mm}$ are considered unacceptable as they lead to overestimation or underestimation of the root canals $[1,9]$.

Table 1. Distance ( $\mathrm{mm}$ ) from the electronic measurements.

\begin{tabular}{ccccccc}
\hline & \multicolumn{2}{c}{ EL APEX $(\mathbf{n = 4 0})$} & \multicolumn{2}{c}{ EL 0.5 $(\mathbf{n = 4 0 )}$} & \multicolumn{2}{c}{ AARL 0.5 $(\mathbf{n = 4 0 )}$} \\
\cline { 2 - 7 } & Mean & S.D & Mean & S.D & Mean & S.D \\
\hline DZ group & 14.38 & 0.27 & 14.38 & 0.30 & 14.21 & 0.20 \\
ES group & 14.36 & 0.24 & 14.20 & 0.24 & 14.26 & 0.26 \\
\hline
\end{tabular}

Table 2: Distribution and percentage of the difference between the Electronic lengths (ELs) and the actual length (AL) obtained at the APEX and 0.5mm mark of the tested devices for Apex locator function.

\begin{tabular}{lcccccccc}
\hline & \multicolumn{3}{c}{ APEX } & \multicolumn{3}{c}{$\mathbf{0 . 5 m m}$} \\
\cline { 2 - 10 } & \multicolumn{2}{c}{ ES group } & \multicolumn{2}{c}{ DZ group } & \multicolumn{2}{c}{ ES group } & \multicolumn{2}{c}{ DZ group } \\
\hline EL-AL & $\mathrm{n}$ & $\%$ & $\mathrm{n}$ & $\%$ & $\mathrm{n}$ & $\%$ & $\mathrm{n}$ & $\%$ \\
$<-\mathbf{- 1 . 5 1}$ & 0 & 0 & 0 & 0 & 0 & 0 & 0 & 0 \\
$\mathbf{- 1 . 5}$ to $\mathbf{1 . 0 1}$ & 0 & 0 & 0 & 5 & 0 & 0 & 0 & 0 \\
$\mathbf{- 1}$ to $\mathbf{- 0 . 5 1}$ & 2 & 5 & 2 & 95 & 9 & 22.5 & 5 & 12.5 \\
$\mathbf{- 0 . 5}$ to $\mathbf{0 . 0 0}$ & 38 & 95 & 38 & 0 & 31 & 77.5 & 35 & 87.5 \\
$\mathbf{- 0 . 0 1}$ to $-\mathbf{0 . 5}$ & 0 & 0 & 0 & 0 & 0 & 0 & 0 & 0 \\
$\mathbf{0 . 5 1}$ to 1 & 0 & 0 & 0 & 0 & 0 & 0 & 0 & 0 \\
$>\mathbf{1 . 0 1}$ & 0 & 0 & 0 & 0 & 0 & 0 & 0 & 0 \\
\hline
\end{tabular}




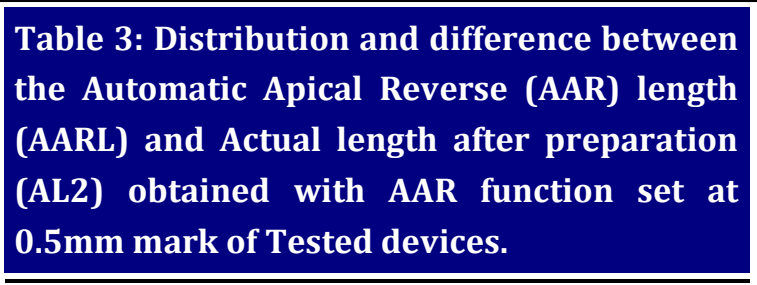

\begin{tabular}{lllll}
\hline & \multicolumn{2}{l}{ DZ group } & \multicolumn{2}{c}{ ES group } \\
\hline AARL-AL2 & $\mathrm{n}$ & $\%$ & $\mathrm{n}$ & $\%$ \\
-1.5 TO -1.01 & 0 & 0 & 0 & 0 \\
-1 TO -0.51 & 2 & 5 & 4 & 10 \\
-0.5 TO 0.00 & 37 & 92.5 & 34 & 85 \\
0.01 TO 0.5 & 1 & 2.5 & 2 & 5 \\
\hline
\end{tabular}

For the EAL function, no overextensions were seen with both the tested devices. In the present study, a slight reduction in length after preparation (AL2) was observed, most probably due to the use of straight canals, and the actual length was obtained after cervical preflaring. The AL2 was measured to prevent bias from the alterations after the preparation of the root canals. In the present study entire preparation of the canals was controlled by AAR function set at $0.5 \mathrm{~mm}$ mark, allowing reversing back after reaching the apical limit. In the literature, after shaping procedure, the apical limit is considered adequate with distance ranging from $0-1 \mathrm{~mm}$ short of AF. In this study, the AAR function set at $0.5 \mathrm{~mm}$ mark provided an acceptable apical limit. The acceptable apical limit seen with apex locator alone was 95\% with the ES group and 97.5\% with DZ group. Over instrumentation noticed with Econnect S apex locator in 5\% of cases and 2.5\% with Dentaport ZX apex locator. This might be due to the manual dexterity of the operator while placing the instrument (F2) manually into the canal after preparation. No incidence of under instrumentation was seen.

\section{Conclusion}

Under the in vitro conditions of this study, both Electronic Apex Locators (i.e., Dentaport ZX and Econnect S) showed an acceptable determination of working length within range $\pm 0.5 \mathrm{~mm}$ from the apex. The AAR function set at the $0.5 \mathrm{~mm}$ mark of Endodontic rotary motor integrated apex locator (E Connect S) provided an adequate apical limit.

Conflicts of interest: Authors declared no conflicts of interest.

Financial support: None

\section{References}

1. Ricucci D, Langeland K. Apical limit of root canal instrumentation and obturation, part 2. A histological study. Int Endod J 1998;31:394-409.

2. Nekoofar et al., The Fundamental Operating Principles of Electronic Root Canal Length Measuring Devices. Int Endodont J. 2006; 39: 595-609.

3. A.K. Ebrahim, R. Wadachi and H. Suda. Electronic Apex Locators - A Review. J Med Dent Sci. 2007; 54,125-136.

4. Vasconcelos BC, Frota LM, de Abreu Souza T, et al. Evaluation of the maintenance of the apical limit during instrumentation with hybrid equipment in rotary and reciprocating modes. J Endo 2015; 41: 682-5.

5. Kobayashi C. Electronic canal length measurement. Oral Surg Oral Med Oral Pathol Oral Radiol Endod 1995;79:226-31.

6. Piasecki L, Carneiro E, da Silva Neto UX, et al. The use of micro-computed tomography to determine the accuracy of 2 electronic apex locators and anatomic variations affecting their precision. J Endod 2016;42:1263-7.

7. Baldi JV, Victorino FR, Bernardes RA, de Moraes IG, Bramante CM, Garcia RB, et al. Influence of embedding media on the assessment of electronic apex locators. J Endod 2007;33:476-9.

8. Camargo EJ, Zapata RO, Medeiros PL, et al. Influence of preflaring on the accuracy of length determination with 4 electronic apex locators. J Endod 2009;35:1300-2.

9. Wu MK, Wesselink PR, Walton RE. Apical terminus location of root canal treatment procedures. Oral Surg Oral Med Oral Pathol Oral Radiol Endod 2000;89:99-103.

10. Vasconcelos BC, Bastos LM, Oliveira AS, et al. Changes in root canal length determined during mechanical preparation stages and their relationship with the accuracy of Root ZX II. J Endodont 2016;42:1683-6. 